\title{
3D Diffraction of obliquely incident SH waves by twin infinitely long cylindrical cavities in layered poroelastic half-space
}

\author{
Jianwen Liang $\cdot$ Bing Han $\cdot$ Zhenning Ba
}

Received: 8 May 2013/Accepted: 24 November 2013/Published online: 3 January 2014

(C) The Seismological Society of China, Institute of Geophysics, China Earthquake Administration and Springer-Verlag Berlin Heidelberg 2013

\begin{abstract}
This paper studies three-dimensional diffraction of obliquely incident plane SH waves by twin infinitely long cylindrical cavities in layered poroelastic half-space using indirect boundary element method. The approach is validated by comparison with the literature, and the effects of cavity interval, incident frequency, and boundary drainage condition on the diffraction are studied through numerical examples. It is shown that, the interaction between two cavities is significant and surface displacement peaks become large when two cavities are close, and the surface displacement may be significantly amplified by twin cavities, and the influence range with large amplification can be as wide as 40 times of the cavity radius. Surface displacements in dry poroelastic case and saturated poroelastic cases with drained and undrained boundaries are evidently different under certain circumstances, and the differences may be much larger than those in the free-field response.
\end{abstract}

Keywords Diffraction - Infinitely long cylindrical cavities - Obliquely incidence - Layered poroelastic half-space $\cdot$ Indirect boundary element method (IBEM) - Amplification · Plane SH waves

\section{Introduction}

Diffraction of elastic waves around underground cavities is an important topic in engineering seismology, however, up

\footnotetext{
J. Liang $\cdot$ B. Han $\cdot$ Z. Ba

Department of Civil Engineering, Tianjin University,

Tianjin 300072, China

J. Liang $(\bowtie) \cdot$ B. Han $\cdot$ Z. Ba

Tianjin Key Laboratory of Civil Engineering Structures \& New

Materials, Tianjin 300072, China

e-mail: liang@tju.edu.cn
}

to now, most studies are limited to either elastic homogeneous half-space or plane strain model. In general, halfspace is layered, and dynamic characteristics of soil layers have significant effect on the wave diffraction (Liang and $\mathrm{Ba}$ 2012); there is necessarily an angle between wave propagation direction and cylindrical cavity axis (De Barros and Luco 1993; Stamos and Beskos 1996; Liang et al. 2012, 2013), or say, the problem is three-dimensional and cannot be simply decomposed into in-plane problem and out-ofplane problem (Liang et al. 2012). Also, in coastal plain or alluvial plain, soil layers below water level are often saturated poroelastic, and pore liquid has significant effects on wave diffraction. However, there are few studies for diffraction of obliquely incident plane waves around infinitely long cylindrical cavities in layered poroelastic half-space.

The purpose of this study is to investigate 3D diffraction of obliquely incident plane SH waves by twin infinitely long cylindrical cavities in layered poroelastic half-space using indirect boundary element method (IBEM), extending the study of single cylindrical cavity in layered elastic half-space (Liang et al. 2012, 2013) to the case of twin cavities and to the case of poroelasticity.

\section{Method}

Figure 1 shows the model of twin infinitely long cylindrical cavities in poroelastic soil layers over elastic half-space (bedrock) for incident plane SH waves, with (a) the view in $x-y$ plane, (b) the view in $y^{\prime}-z$ plane, and (c) the crosssection of the cavities in $x-z$ plane. The angle with $y$ in $x-y$ plane is $\theta_{\mathrm{h}}$, and the angle with $y^{\prime}$ in $y^{\prime}-z$ plane is $\theta_{\mathrm{v}}$. The thickness of the soil layers is $H$, the radius and buried depth of the cavity is $a$ and $h$, and the distance between two cavities is $S$. The boundary of two cavities is described by $\Gamma$. 


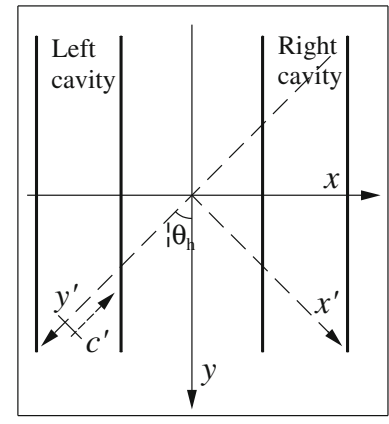

(a)

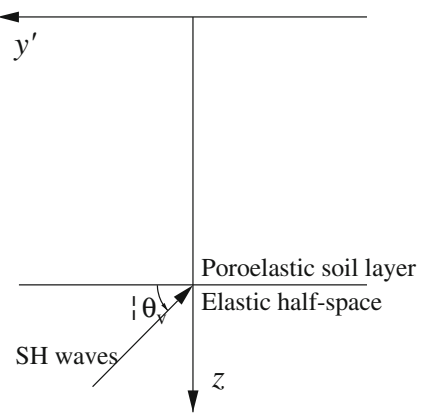

(b)

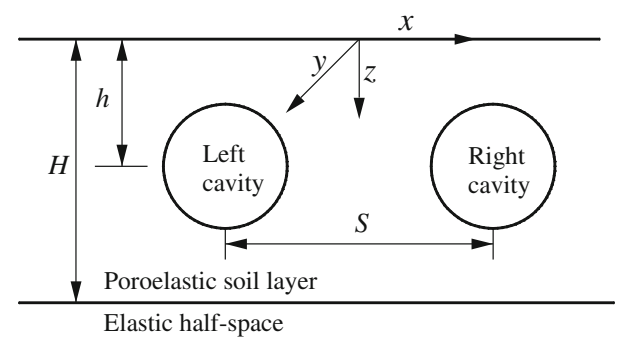

(c)

Fig. 1 The model of twin cylindrical cavities in poroelastic soil layers over elastic half-space (bedrock) for incident plane SH waves, with a the view in $x-y$ plane, $\mathbf{b}$ the view in $y^{\prime}-z$ plane and $\mathbf{c}$ the cross-section of the cavities in $x-z$ plane. $\theta_{\mathrm{h}}$ is the angle with $y$ in $x-y$ plane, and $\theta_{\mathrm{v}}$ is the angle with $y^{\prime}$ in $y^{\prime}-z$ plane, $H$ is the thickness of the soil layers, $h$ is the buried depth of the cavity, and $S$ is the distance between two cavities

The cylindrical cavities are infinitely long, and the wave fields at two different cross-sections perpendicular to the cavity axis are identical but shifted in time, therefore, the wave field can be represented by the dynamic response of moving loads. This allows only one cross-section of the cavities to be used for calculation. The IBEM is used to determine the wave diffraction around cavities in poroelastic soil layers over elastic half-space. The free-field response is calculated to determine the dynamic response at the surface of the fictitious cavities. Moving distributed loads and pore pressure is applied on the surface of the fictitious cavities to calculate the Green's functions for the dynamic responses at the surface of the fictitious cavities. The moving distributed load and pore pressure are determined by boundary conditions. Finally, the solution is obtained by adding the dynamic responses of the free field and the response due to the moving distributed loads.

\subsection{Biot's theory}

The displacement of the solid frame and the fluid displacement relative to the solid frame are denoted by $u_{i}$ and $w_{i}(i=x, y)$, respectively, then the constitutive relationship (Biot 1941) of a homogeneous poroelastic medium can be expressed as

$\sigma_{i j}=2 \mu \varepsilon_{i j}+\lambda \delta_{i j} e-\alpha \delta_{i j} p ; \quad i, j=x, y$

$p=-\alpha M u_{i, i}-M w_{i, i}$

where $\sigma_{i j}$ is the total stress component of the bulk material; $\varepsilon_{i j}$ and $e$ are strain components and dilatation of the solid frame, respectively; $\lambda$ and $\mu$ are Lame constants of the bulk material; $p$ is pore pressure; $\delta_{i j}$ is Kronecker delta; and $\alpha$ and $M$ are Biot's parameters describing compressibility of the two-phased material and $0 \leq \alpha \leq 1$ and $0 \leq M<\infty$.

The equations of motion (Biot 1962) of a homogeneous poroelastic medium can be expressed in terms of displacements $u_{i}$ and $w_{i}$ as

$$
\begin{aligned}
& \mu u_{i, j j}+\left(\lambda+\alpha^{2} M+\mu\right) u_{j, j i}+\alpha M w_{j, j i}=\rho \ddot{u}_{i}+\rho_{\mathrm{f}} \ddot{w}_{i} \\
& \alpha M u_{j, j i}+M w_{j, j i}=\rho_{\mathrm{f}} \ddot{u}_{i}+m \ddot{w}_{i}+b \dot{w}_{i}
\end{aligned}
$$

where $\rho=(1-n) \rho_{\mathrm{s}}+n \rho_{\mathrm{f}}, \rho_{\mathrm{s}}$ and $\rho_{\mathrm{f}}$ are mass density of the solid grain and the pore fluid, respectively; $n$ is porosity of the solid frame; $b$ is a parameter that accounts for the internal friction due to the relative motion between the solid frame and the pore fluid, and $b=0$ if the internal friction is neglected; and $m$ is a density-like parameter depending on $\rho_{\mathrm{f}}$ and geometry of the pores.

\subsection{Free-field response}

The free-field response of layered poroelastic half-space can be calculated by direct stiffness method, to determine the 3D dynamic response on the boundary of the fictitious twin cavities and on the surface of the half-space.

For a saturated poroelastic layer, the displacement amplitudes and the external load amplitudes at the top and bottom of the layer can be expressed by the amplitude coefficients of the up-going waves and of the down-going waves in wave-number domain, i.e.,

$$
\begin{aligned}
& \left\{U_{x 1}, U_{y 1}, i U_{z 1}, i w_{z 1}, U_{x 2}, U_{y 2}, i U_{z 2}, i w_{z 2}\right\}^{\mathrm{T}} \\
& \quad=S_{1}\left\{A_{P 1}, B_{P 1}, A_{P 2}, B_{P 2}, A_{\mathrm{SV}}, B_{\mathrm{SV}}, A_{\mathrm{SH}}, B_{\mathrm{SH}}\right\}^{\mathrm{T}} \\
& \left\{Q_{x 1}, Q_{y 1}, i Q_{z 1}, i Q_{f 1}, Q_{x 2}, Q_{y 2}, i Q_{z 2}, i Q_{f 2}\right\}^{\mathrm{T}} \\
& \quad=S_{2}\left\{A_{P 1}, B_{P 1}, A_{P 2}, B_{P 2}, A_{\mathrm{SV}}, B_{\mathrm{SV}}, A_{\mathrm{SH}}, B_{\mathrm{SH}}\right\}^{\mathrm{T}}
\end{aligned}
$$

where, $U_{x 1}, U_{y 1}, U_{z 1}$, and $w_{z 1}$ are the displacement amplitudes at the top of the layer, $U_{x 2}, U_{y 2}, U_{z 2}$, and $w_{z 2}$ are the displacement amplitudes at the bottom of the layer, $Q_{x 1}$, $Q_{y 1}, Q_{z 1}$, and $Q_{f 1}$ are the external loads at the top of the layer, and $Q_{x 2}, Q_{y 2}, Q_{z 2}$, and $Q_{f 2}$ are the external loads at the bottom of the layer, and $A_{P 1}, B_{P 1}, A_{P 2}, B_{P 2}, A_{\mathrm{SV}}, B_{\mathrm{SV}}$, $A_{\mathrm{SH}}$, and $B_{\mathrm{SH}}$ are the amplitude coefficients of the up-going waves and of the down-going waves, and 
$\boldsymbol{S}_{\mathbf{1}}=\left[\begin{array}{cccccccc}l_{x 1} & l_{x 1} & l_{x 2} & l_{x 2} & -m_{x} m_{z} \beta & m_{x} m_{z} \beta & -m_{y} \beta & -m_{y} \beta \\ l_{y 1} & l_{y 1} & l_{y 2} & l_{y 2} & -m_{y} m_{z} \beta & m_{y} m_{z} \beta & m_{x} \beta & m_{x} \beta \\ -i l_{z 1} & i l_{z 1} & -i l_{z 2} & i l_{z 2} & -i / \beta & -i / \beta & 0 & 0 \\ -i D_{1} l_{z 1} & i D_{1} l_{z 1} & -i D_{2} l_{z 2} & i D_{2} l_{z 2} & -i D_{3} / \beta & -i D_{3} / \beta & 0 & 0 \\ l_{x 1} \beta_{1} & l_{x 1} \beta_{2} & l_{x 2} \beta_{3} & l_{x 2} \beta_{4} & -m_{x} m_{z} \beta \beta_{5} & m_{x} m_{z} \beta \beta_{6} & -m_{y} \beta \beta_{5} & -m_{y} \beta \beta_{6} \\ l_{y 1} \beta_{1} & l_{y 1} \beta_{2} & l_{y 2} \beta_{3} & l_{y 2} \beta_{4} & -m_{y} m_{z} \beta \beta_{5} & m_{y} m_{z} \beta \beta_{6} & m_{x} \beta & m_{x} \beta \\ -i l_{z 1} \beta_{1} & i l_{z 1} \beta_{2} & -i l_{z 2} \beta_{3} & i l_{z 2} \beta_{4} & -i \beta_{5} / \beta & -i \beta_{6} / \beta & 0 & 0 \\ -i D_{1} l_{z 1} \beta_{1} & i D_{1} l_{z 1} \beta_{2} & -i D_{2} l_{z 2} \beta_{3} & i D_{2} l_{z 2} \beta_{4} & -i D_{3} \beta_{5} / \beta & -i D_{3} \beta_{6} / \beta & 0 & 0\end{array}\right]$

$\boldsymbol{S}_{2}=\left[\begin{array}{cccccccc}C_{11} & C_{12} & C_{13} & C_{14} & C_{15} & C_{16} & C_{17} & C_{18} \\ C_{21} & C_{22} & C_{23} & C_{24} & C_{25} & C_{26} & C_{27} & C_{28} \\ C_{31} & C_{32} & C_{33} & C_{34} & C_{35} & C_{36} & 0 & 0 \\ C_{41} & C_{42} & C_{43} & C_{44} & 0 & 0 & 0 & 0 \\ C_{11} \beta_{1} & C_{12} \beta_{2} & C_{13} \beta_{3} & C_{14} \beta_{4} & C_{15} \beta_{5} & C_{16} \beta_{6} & C_{17} \beta_{5} & C_{18} \beta_{6} \\ C_{21} \beta_{1} & C_{22} \beta_{2} & C_{23} \beta_{3} & C_{24} \beta_{4} & C_{25} \beta_{5} & C_{26} \beta_{6} & C_{27} \beta_{5} & C_{28} \beta_{6} \\ C_{31} \beta_{1} & C_{32} \beta_{2} & C_{33} \beta_{3} & C_{34} \beta_{4} & C_{35} \beta_{5} & C_{36} \beta_{6} & 0 & 0 \\ C_{41} \beta_{1} & C_{42} \beta_{2} & C_{43} \beta_{3} & C_{44} \beta_{4} & 0 & 0 & 0 & 0\end{array}\right]$

where,

$\beta_{1}=\exp \left(i k_{p 1 z} z\right), \beta_{2}=\exp \left(-i k_{p 1 z} z\right), \beta_{3}=\exp \left(i k_{p 2 z} z\right), \beta_{4}=\exp \left(-i k_{p 2 z} z\right)$,

$\beta_{5}=\exp \left(i k_{s z} z\right), \beta_{6}=\exp \left(-i k_{s z} z\right), \beta=1 / \sqrt{m_{x}^{2}+m_{y}^{2}}, C_{11}=-2 i \mu^{*} l_{z 1} k_{x}, C_{12}=2 i \mu^{*} l_{z 1} k_{x}$,

$C_{13}=-2 i \mu^{*} l_{z 2} k_{x}, C_{14}=2 i \mu^{*} l_{z 2} k_{x}, C_{15}=i \mu^{*} \beta\left(m_{x} m_{z} k_{s z}-k_{x} m_{x}^{2}-k_{x} m_{y}^{2}\right)$,

$C_{16}=i \mu^{*} \beta\left(m_{x} m_{z} k_{s z}-k_{x} m_{x}^{2}-k_{x} m_{y}^{2}\right), C_{17}=i m_{y} k_{s z} \mu^{*} \beta, C_{18}=-i m_{y} k_{s z} \mu^{*} \beta$,

$C_{21}=-2 i \mu^{*} l_{z 1} k_{y}, C_{22}=2 i \mu^{*} l_{z 1} k_{y}, C_{23}=-2 i \mu^{*} l_{z 2} k_{y}, C_{24}=2 i \mu^{*} l_{z 2} k_{y}$,

$C_{25}=i \mu^{*} \beta\left(m_{y} m_{z} k_{s z}-k_{y} m_{x}^{2}-k_{y} m_{y}^{2}\right), C_{26}=i \mu^{*} \beta\left(m_{y} m_{z} k_{s z}-k_{y} m_{x}^{2}-k_{y} m_{y}^{2}\right)$,

$C_{27}=-i m_{x} k_{s z} \mu^{*} \beta, C_{28}=i m_{x} k_{s z} \mu^{*} \beta, C_{31}=-\left(l_{x 1} k_{x}+l_{y 1} k_{y}+l_{z 1} k_{p 1 z}\right)\left(\lambda^{*}+\alpha M\left(D_{1}+\alpha\right)\right)-2 \mu^{*} l_{z 1} k_{p 1 z}$,

$C_{32}=-\left(l_{x 1} k_{x}+l_{y 1} k_{y}+l_{z 1} k_{p 1 z}\right)\left(\lambda^{*}+\alpha M\left(D_{1}+\alpha\right)\right)-2 \mu^{*} l_{z 1} k_{p 1 z}$

$C_{33}=-\left(l_{x 2} k_{x}+l_{y 2} k_{y}+l_{z 2} k_{p 2 z}\right)\left(\lambda^{*}+\alpha M\left(D_{2}+\alpha\right)\right)-2 \mu^{*} l_{z 2} k_{p 2 z}$,

$C_{34}=-\left(l_{x 2} k_{x}+l_{y 2} k_{y}+l_{z 2} k_{p 2 z}\right)\left(\lambda^{*}+\alpha M\left(D_{2}+\alpha\right)\right)-2 \mu^{*} l_{z 2} k_{p 2 z}, C_{35}=-2 \mu^{*} k_{s z} / \beta, C_{36}=2 \mu^{*} k_{s z} / \beta$,

$C_{41}=-M\left(D_{1}+\alpha\right)\left(l_{x 1} k_{x}+l_{y 1} k_{y}+l_{z 1} k_{p 1 z}\right), C_{42}=-M\left(D_{1}+\alpha\right)\left(l_{x 1} k_{x}+l_{y 1} k_{y}+l_{z 1} k_{p 1 z}\right)$,

$C_{43}=-M\left(D_{2}+\alpha\right)\left(l_{x 2} k_{x}+l_{y 2} k_{y}+l_{z 2} k_{p 2 z}\right), C_{44}=-M\left(D_{2}+\alpha\right)\left(l_{x 2} k_{x}+l_{y 2} k_{y}+l_{z 1} k_{p 2 z}\right)$,

$\lambda^{*}=\lambda\left(1+2 i \zeta^{L}\right), \mu^{*}=\mu\left(1+2 i \zeta^{L}\right), m_{x}=c_{s}^{L} k_{x} / w, m_{y}=c_{s}^{L} k_{y} / w, m_{z}=\sqrt{1.0-m_{x}^{2}-m_{y}^{2}}$,

$l_{x 1}=c_{p 1}^{L} k_{x} / w, l_{x 2}=c_{p 2}^{L} k_{x} / w, l_{y 1}=c_{p 1}^{L} k_{y} / w, l_{y 2}=c_{p 2}^{L} k_{y} / w, l_{z 1}=\sqrt{1.0-l_{x 1}^{2}-l_{y 1}^{2}}$,

$l_{z 2}=\sqrt{1.0-l_{x 2}^{2}-l_{y 2}^{2}}, k_{p j z}=l_{z j} \omega / c_{p j}^{L}(j=1,2), k_{s z}=m_{z} \omega / c_{s}^{L}$,

$D_{i}=\left(\lambda_{c}+2 \mu-\rho c_{p i}^{L 2}\right) /\left(\rho_{f} c_{p i}^{L 2}-\alpha M\right)(i=1,2), D_{3}=\rho_{f} \omega^{2} /\left(i b \omega-m \omega^{2}\right)$, 
where, $z$ is thickness of the soil layer; $\omega$ is circular frequency of incident waves; $k_{x}$ and $k_{y}$ are two horizontal wave numbers; $k_{s z}, k_{p 1 z}$, and $k_{p 2 z}$ are wave numbers in vertical direction for $\mathrm{S}$ waves and two $\mathrm{P}$ waves, respectively; $c_{s}^{L}, c_{p 1}^{L}$, and $c_{p 2}^{L}$ are velocities for $\mathrm{S}$ waves and two $\mathrm{P}$ waves in the soil layer, respectively; $m_{x}, m_{y}$, and $m_{z}$ are parameters related to the propagation direction for $\mathrm{S}$ waves; $l_{x 1}, l_{y 1}$, and $l_{z 1}$ and $l_{x 2}, l_{y 2}$, and $l_{z 2}$ are those for two $\mathrm{P}$ waves, respectively; $\lambda_{c}=\lambda+\alpha^{2} M$; and $\zeta^{L}$ is damping ratio for the soil layer.

Eliminating the amplitudes of the up-going and downgoing waves can result in the dynamic stiffness matrix $S_{\mathbf{P 1}-\mathbf{P 2}-\mathbf{S V}-\mathbf{S H}}^{\mathbf{L}}$ of the saturated poroelastic soil layer,

$S_{\mathrm{P} 1-\mathrm{P} 2-\mathrm{SV}-\mathrm{SH}}^{\mathrm{L}}=S_{2} S_{1}^{-1}$

The dynamic stiffness matrix $S_{\mathbf{P 1}-\mathbf{P 2}-\mathbf{S V}-\mathbf{S H}}^{\mathbf{R}}$ of saturated poroelastic half-space can be obtained by applying loads at the free surface of the saturated poroelastic half-space and by considering only the down-going waves, which satisfies the radiation condition that without energy propagating from infinity toward the free surface. Letting $A_{P 1}=A_{P 2}=A_{\mathrm{SV}}=A_{\mathrm{SH}}=0$ in Eqs. (5) and (6) and eliminating $B_{P 1}, B_{P 2}, B_{\mathrm{SV}}$, and $B_{\mathrm{SH}}$, the saturated poroelastic half-space matrix $\mathbf{S}_{P 1-P 2-\mathrm{SV}-\mathrm{SH}}^{R}$ is obtained.

Introducing the continuity of displacements and the equilibrium of stresses and pore pressures at the interfaces of the layers, the dynamic stiffness matrix $S_{\mathbf{P 1}-\mathbf{P 2}-\mathbf{S V}-\mathbf{S H}}$ of a layered half-space can be assembled by using the soil layer stiffness matrices $S_{\mathbf{P 1}-\mathbf{P 2}-\mathbf{S V}-\mathbf{S H}}^{\mathrm{L}}$ and the half-space stiffness matrix $S_{\mathbf{P 1} 1 \mathbf{P 2}-\mathbf{S V}-\mathbf{S H}}^{\mathbf{R}}$.

For a layered half-space, the displacements of each layer can be solved by the following equation

$S_{\mathrm{P} 1-\mathrm{P} 2-\mathrm{SV}-\mathrm{SH}} U=Q$

$\boldsymbol{U}$ is the vector of solid frame displacement and of relative displacement between pore liquid and solid frame, and $\boldsymbol{Q}$ is the vector of stress and pore pressure. If we choose the bedrock outcrop as the control point and let $U_{x 0}, U_{y 0}, U_{z 0}$, and $w_{z 0}$ be the bedrock input motion, the last four elements in vector $\boldsymbol{Q}$ can be obtained through

$\left\{\begin{array}{c}R_{x 0} \\ R_{y 0} \\ i R_{z 0} \\ -i R_{f 0}\end{array}\right\}=S_{\mathbf{P 1}-\mathbf{P 2}-\mathbf{S V}-\mathbf{S H}}^{\mathbf{R}}\left\{\begin{array}{c}U_{x 0} \\ U_{y 0} \\ i U_{z 0} \\ i w_{z 0}\end{array}\right\}$

while the other elements of vector $Q$ are zero. Once $U$ is obtained, the amplitude coefficients of the up-going waves and down-going waves in each layer can be determined. Finally we get the displacement $\left\{U_{x}^{\mathrm{f}}, U_{y}^{\mathrm{f}}, U_{z}^{\mathrm{f}}, w_{n}^{\mathrm{f}}\right\}^{\mathrm{T}}$ and stress $\left\{t_{x}^{\mathrm{f}}, t_{y}^{\mathrm{f}}, t_{z}^{\mathrm{f}}, t_{f}^{\mathrm{f}}\right\}^{\mathrm{T}}$ on the boundary of the fictitious twin cavities and on the surface of the half-space for the drained boundary condition, and $\left\{U_{\mathrm{u} x}^{\mathrm{f}}, U_{\mathrm{u} y}^{\mathrm{f}}, U_{\mathrm{u} z}^{\mathrm{f}}, w_{\mathrm{u} n}^{\mathrm{f}}\right\}^{\mathrm{T}}$ and $\left\{t_{\mathrm{u} x}^{\mathrm{f}}, t_{\mathrm{u} y}^{\mathrm{f}}, t_{\mathrm{u} z}^{\mathrm{f}}, t_{\mathrm{u} f}^{\mathrm{f}}\right\}^{\mathrm{T}}$ on the boundary of the fictitious twin cavities and on the surface of the half-space for the undrained boundary condition.

\subsection{Dynamic Green's function}

The dynamic Green's functions of moving distributed loads and pore pressure acting on inclined line in layered saturated poroelastic half-space can be obtained from the extension of the Green's functions of moving distributed loads on inclined line in layered elastic half-space (Liang et al. 2012), combining with Biot's theory of poroelasticity (Biot 1941; Biot 1962).

The Green's functions of distributed loads and pore pressure acting on inclined line in layered saturated poroelastic half-space are deduced, then the moving Green's functions can be obtained by Fourier integration along the moving direction (the axis of the cavities)

$$
\begin{aligned}
\bar{F}(x, y, z)= & \int_{-\infty}^{\infty} \exp \left(i \omega\left(t-\varepsilon / c^{\prime}\right)\right) \\
& \int_{-\infty}^{\infty} \int_{-\infty}^{\infty} F\left(k_{x}, k_{y}, z\right) \exp \left(-i k_{x} x\right) \\
& \times \exp \left(-i k_{y}(y-\varepsilon)\right) \mathrm{d} k_{x} \mathrm{~d} k_{y} \mathrm{~d} \varepsilon \\
= & 2 \pi \int_{-\infty}^{\infty} \int_{-\infty}^{\infty} F\left(k_{x}, k_{y}, z\right) \delta\left(k_{y}^{\prime}-k_{y}\right) \exp \left(-i k_{x} x\right) \\
\times & \exp \left(-i k_{y} y\right) \mathrm{d} k_{x} \mathrm{~d} k_{y} \\
= & 2 \pi \exp \left(-i k_{y}^{\prime} y\right) \int_{-\infty}^{\infty} F\left(k_{x}, k_{y}^{\prime}, z\right) \exp \left(-i k_{x} x\right) \mathrm{d} k_{x}
\end{aligned}
$$

where $\bar{F}(x, y, z)$ is the moving Green's functions in the space domain, $F\left(k_{x}, k_{y}^{\prime}, z\right)$ the dynamic Green's functions in the wave-number domain, $k_{y}^{\prime}=\omega / c^{\prime}, \omega$ is the incident frequency, $c^{\prime}$ is the moving velocity of distributed loads and pore pressure along the axis of the cavities, $c^{\prime}=c_{s}^{R} /\left(\cos \theta_{h} \cos \theta_{v}\right)$, with $c_{s}^{R}$ the shear-wave velocity of the half-space.

To obtain the Green's functions of distributed loads and pore pressure acting on inclined line in layered saturated half-space, the distributed loads and pore pressure are transformed into wave-number domain by the Fourier transform, and the Green's functions in wave-number domain are then obtained. Figure 2 shows the distributed loads and pore pressure acting on an inclined line in a saturated poroelastic layer, with the distributed loads acting on soil frame in the $x$-, $y$-, and $z$-directions. Because only one layer is loaded, two additional interfaces are introduced at the top and bottom boundary of the layer. The loaded layer 

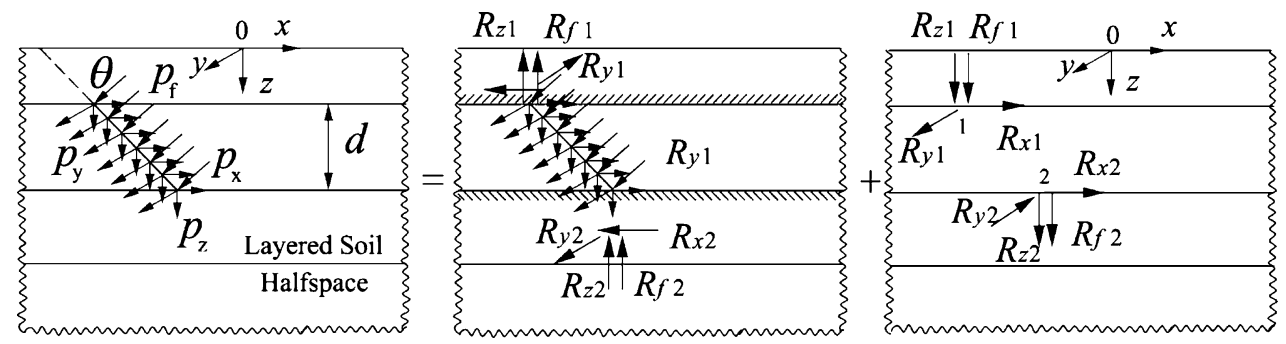

Fig. 2 Distributed loads and pore pressure acting on an inclined line in soil layers

is fixed at the two interfaces, and the corresponding reaction forces $R_{x 1}, R_{y 1}, R_{z 1}, R_{f 1}$ (forces at the top interface of the fixed layer) and $R_{x 2}, R_{y 2}, R_{z 2}, R_{f 2}$ (forces at the bottom interface of the fixed layer) are calculated. The directions of these forces are then reversed, and they are applied as external loads to the system to determine the global response, and the dynamic responses of the system induced by the external forces can be implemented using the direct stiffness method. The whole responses are determined by adding the result of the fixed layer analysis to the result of the reaction forces (Fig. 2). Finally, the Green's functions in the space domain are obtained by inverse Fourier transform. More details about the approach may be found in Liang and You $(2004,2005)$ and Liang et al. (2006).

Let $g_{u}(\Gamma)$ and $g_{t}(\Gamma)$ be the moving Green's functions of displacements and stresses for the case of drained boundary condition, and $g_{\mathrm{u} u}(\Gamma)$ and $g_{\mathrm{u} t}(\Gamma)$ be the moving Green's functions corresponding to the case of undrained boundary condition, the solid frame displacement and the relative displacement between pore liquid and solid frame, and the solid frame stresses and the pore pressure at the cavities boundary $\Gamma$ can be expressed as

$\left\{U_{x}^{p}, U_{y}^{p}, U_{z}^{p}, w_{n}^{p}\right\}^{\mathrm{T}}=g_{u}(\Gamma)\left\{p_{x}, p_{y}, p_{z}, p_{f}\right\}^{\mathrm{T}}$

$\left\{t_{x}^{p}, t_{y}^{p}, t_{z}^{p}, t_{f}^{p}\right\}^{\mathrm{T}}=g_{t}(\Gamma)\left\{p_{x}, p_{y}, p_{z}, p_{f}\right\}^{\mathrm{T}}$

$\left\{U_{x}^{p}, U_{y}^{p}, U_{z}^{p}, w_{n}^{p}\right\}^{\mathrm{T}}=g_{\text {uu }}(\Gamma)\left\{p_{x}, p_{y}, p_{y}, p_{f}\right\}^{\mathrm{T}}$

$\left\{t_{x}^{p}, t_{y}^{p}, t_{z}^{p}, t_{f}^{p}\right\}^{\mathrm{T}}=g_{\mathrm{ut}}(\Gamma)\left\{p_{x}, p_{y}, p_{z}, p_{f}\right\}^{\mathrm{T}}$

where $\left\{U_{x}^{p}, U_{y}^{p}, U_{z}^{p}, w_{n}^{p}\right\}^{\mathrm{T}}$ and $\left\{t_{x}^{p}, t_{y}^{p}, t_{z}^{p}, t_{f}^{p}\right\}^{\mathrm{T}}$ are the results attributable to the moving fictitious distributed loads or pore pressure, and the elements in the vector $\left\{p_{x}, p_{y}, p_{z}, p_{f}\right\}^{\mathrm{T}}$ are the fictitious distributed loads and pore pressure.

\subsection{Boundary conditions}

The moving distributed loads and pore pressure can be solved by boundary conditions at the surface of two cavities.

The drained boundary condition can be written as
$\int_{\Gamma} W(\Gamma)^{\mathrm{T}}\left(\left\{t_{x}^{p}, t_{y}^{p}, t_{z}^{p}, t_{f}^{p}\right\}^{\mathrm{T}}+\left\{t_{x}^{\mathrm{f}}, t_{y}^{\mathrm{f}}, t_{z}^{\mathrm{f}}, t_{f}^{\mathrm{f}}\right\}^{\mathrm{T}}\right) \mathrm{d} \Gamma=0$

for drained case, in which, $W(\Gamma)$ is weighted function, which may be taken as unit matrix.

Similarly, the undrained boundary condition can be written as

$\int_{\Gamma} W(\Gamma)^{\mathrm{T}}\left(\left\{t_{x}^{p}, t_{y}^{p}, t_{z}^{p}, w_{n}^{p}\right\}^{\mathrm{T}}+\left\{t_{x}^{\mathrm{f}}, t_{y}^{\mathrm{f}}, t_{z}^{\mathrm{f}}, w_{n}^{\mathrm{f}}\right\}^{\mathrm{T}}\right) \mathrm{d} \Gamma=0$

Then the displacement in layered half-space for drained case can be obtained by

$\left\{\begin{array}{l}U_{x}(x, z) \\ U_{y}(x, z) \\ U_{z}(x, z)\end{array}\right\}=\left\{\begin{array}{l}U_{x}^{\mathrm{f}}(x, z) \\ U_{y}^{\mathrm{f}}(x, z) \\ U_{z}^{\mathrm{f}}(x, z)\end{array}\right\}+\left[g_{u}(x, z)\right] T_{p}^{-1} T_{\mathrm{f}}$

where, $T_{p}=\int_{\Gamma} W(\Gamma)^{T} g_{t}(\Gamma) \mathrm{d} \Gamma$ and $T_{\mathrm{f}}=\int_{\Gamma} W(\Gamma)^{T} t^{\mathrm{f}}(\Gamma) \mathrm{d} \Gamma$, $t^{\mathrm{f}}(\Gamma)=\left\{t_{x}^{\mathrm{f}}, t_{y}^{\mathrm{f}}, t_{z}^{\mathrm{f}}, t_{f}^{\mathrm{f}}\right\}^{T}$.

Similarly, the displacement in layered half-space for undrained boundary condition can be written as

$\left\{\begin{array}{l}U_{x}(x, z) \\ U_{y}(x, z) \\ U_{z}(x, z)\end{array}\right\}=\left\{\begin{array}{c}U_{x}^{\mathrm{f}}(x, z) \\ U_{y}^{\mathrm{f}}(x, z) \\ U_{z}^{\mathrm{f}}(x, z)\end{array}\right\}+\left[g_{u t}(x, z)\right] T_{p}^{-1} T_{\mathrm{f}}$

where, $T_{p}=\int_{\Gamma} W(\Gamma)^{T} g_{u t}(\Gamma) \mathrm{d} \Gamma$ and $T_{\mathrm{f}}=\int_{\Gamma} W(\Gamma)^{T} t^{\mathrm{f}}(\Gamma) \mathrm{d} \Gamma$, $t^{\mathrm{f}}(\Gamma)=\left\{t_{x}^{\mathrm{f}}, t_{y}^{\mathrm{f}}, t_{z}^{\mathrm{f}}, w_{n}^{\mathrm{f}}\right\}$.

\section{Verification of accuracy}

To verify accuracy of the method, Fig. 3 shows the comparison between the degenerated solutions of this study and those by De Barros and Luco (1993) for surface displacement around single infinitely long cylindrical cavity in homogeneous elastic half-space subjected to incident plane SH waves. The cavity is featured with buried depth $d /$ $a=2$, where $a$ is the radius of the cavity. A dimensionless frequency $\eta=2 a / \lambda$ is defined, where $\lambda$ is the wavelength 

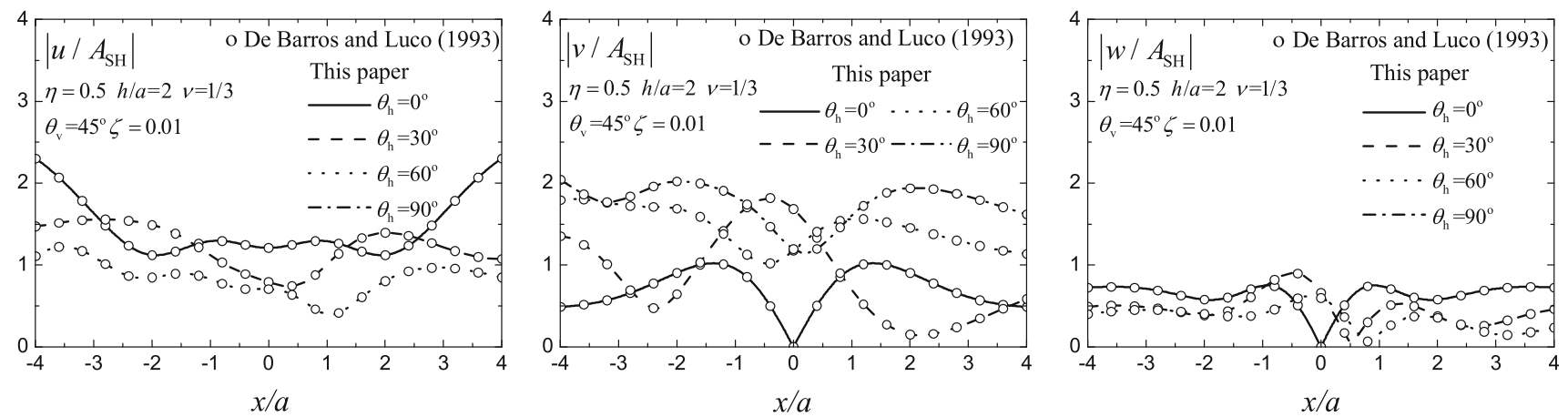

Fig. 3 Comparison with de Barros and Luco (1993) for dynamic response of single cavity in homogeneous elastic half-space
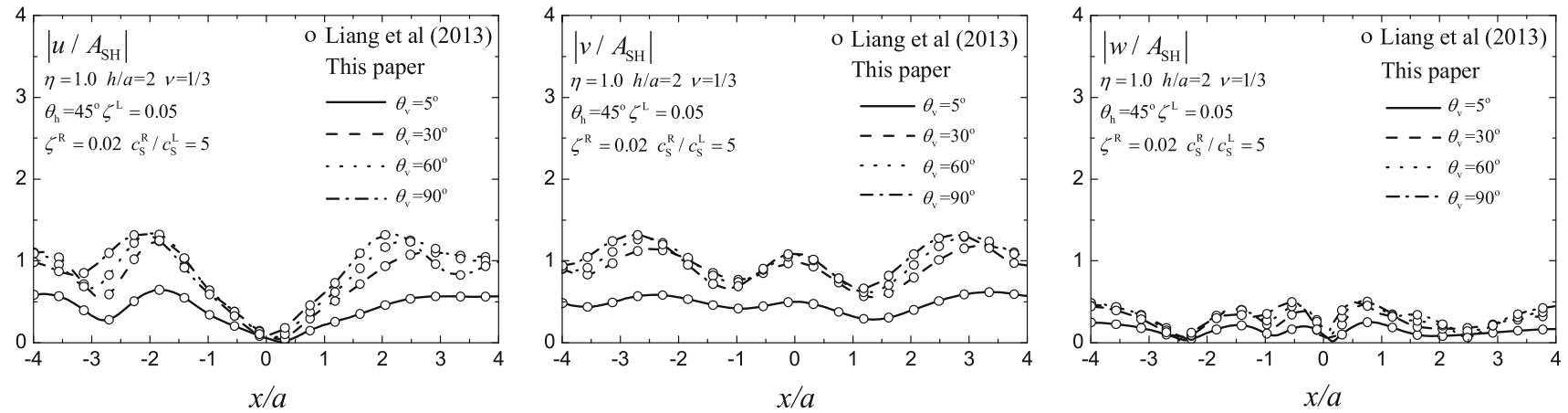

Fig. 4 Comparison with Liang et al. (2013) for dynamic response of single cavity in layered elastic half-space

of shear wave in the half-space, and $\eta=0.5$. Other parameters are as follows: Poisson's ratio $v=1 / 3$ and damping ratio $\xi=0.01$. It is shown that two solutions are in excellent agreement.

Figure 4 shows the comparison between the degenerated solutions of this study and those by Liang et al. (2013) for surface displacement around single infinitely long cylindrical cavity in single elastic soil layer over elastic bedrock half-space, subjected to incident plane SH waves. The ratio of soil layer thickness to cavity radius $h / a=4$, and other parameters are described in the figure. It is shown that two solutions are identical.

\section{Numerical results and discussion}

Figures 5, 6, 7, 8, and 9 illustrate surface displacements versus $x / a$ around twin infinitely long cylindrical cavities in the case of dry poroelastic soil layer and in the cases of saturated poroelastic soil layer with drained and undrained boundaries, respectively, and in all three cases the bedrock half-space is elastic. For the purpose of amplification evaluation of incident waves of different frequencies, Fig. 10 shows spectral amplification of the surface displacements of the free field.
The parameters for the model are as follows: $H / a=4$, $h / a=2, \theta_{\mathrm{h}}=\theta_{\mathrm{v}}=45^{\circ}, S / a=2.5,5,10$, and 20 . The dimensionless frequency is $\eta=2 a / \lambda=0.125,0.25,0.5$, 1.0, and 2.0, where $\lambda$ is the wavelength of shear wave in poroelastic soil layer. The parameters for soil layer are as follows: porosity of the solid frame $n^{\mathrm{L}}=0.3$, shear modulus of the solid frame $\mu^{\mathrm{L}}=216 \mathrm{MPa}$, mass density of the solid grain $\rho_{\mathrm{s}}=2400 \mathrm{~kg} / \mathrm{m}^{3}$, Poisson's ratio $=0.25$, mass density of the pore liquid $\rho_{\mathrm{f}}=1000 \mathrm{~kg} / \mathrm{m}^{3}$, damping ratio $\zeta^{\mathrm{L}}=0.05$, and other Biot's parameters $b=0$, $M=2490 \mathrm{MPa}, m=7222 \mathrm{~kg} / \mathrm{m}^{3}$, and $\alpha=0.8287$. The parameters for elastic bedrock half-space are as follows: shear modulus $\mu^{\mathrm{R}}=5400 \mathrm{MPa}$, mass density $\rho^{\mathrm{R}}=$ $2400 \mathrm{~kg} / \mathrm{m}^{3}$, Poisson's ratio $v^{R}=0.25$, and damping ratio $\zeta^{\mathrm{R}}=0.02$.

It can be seen that, the surface displacement peaks appear around twin cavities; when $x / a$ becomes large, or the observation points are far from twin cavities, the surface displacement approach to the free-field response. When two cavities are close, there is only one group of displacement peaks, and the displacement peaks become larger, which is due to the interaction between two cavities; while the cavity interval increases, the displacement peaks gradually change into two groups with each corresponding to one cavity, and the displacement peaks then become smaller. It can be seen 

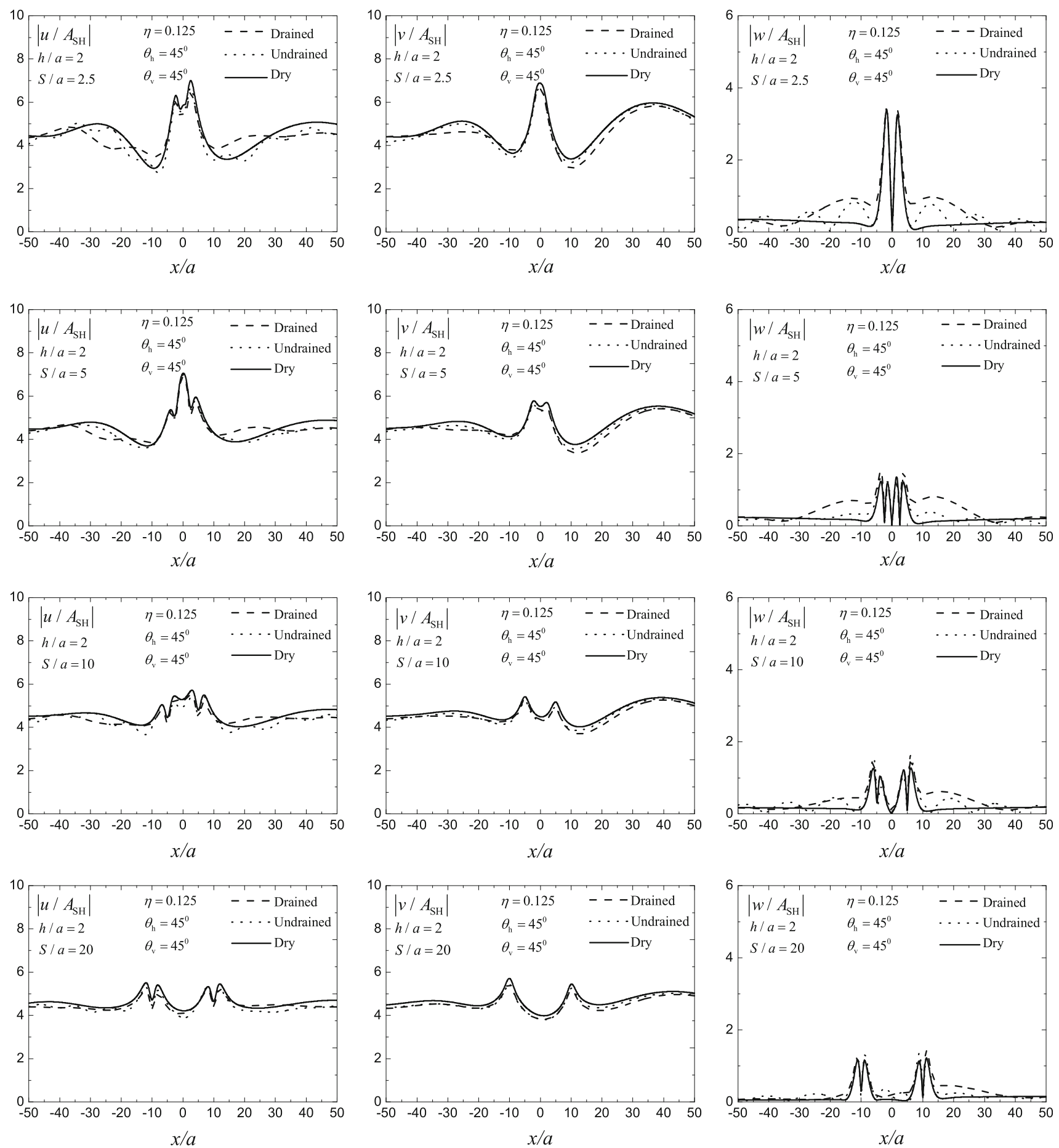

Fig. 5 Surface displacement around twin cavities in the case of dry poroelastic soil layer, the cases of saturated poroelastic soil layer with drained and undrained boundaries for $\eta=0.125$

that the interaction between two cavities depends on the cavity interval and incident frequency.

It may be concluded from these figures that, there is significant interaction between two cavities, and the interaction amplifies the surface dynamic response tremendously, and the influence range with large amplification can be as wide as $40( \pm 20)$ times of the cavity radius.
It can be found that, the surface displacements in both two horizontal directions are extremely large for $\eta=0.125$, and the horizontal displacement peaks in $x$ direction are up to 7.00 (for $S / a=2.5$ ) and 7.05 (for $S /$ $a=5$ ), because the incident frequency is equal to the first resonant frequency of the free field, and the free-field response is large (Fig. 10). 

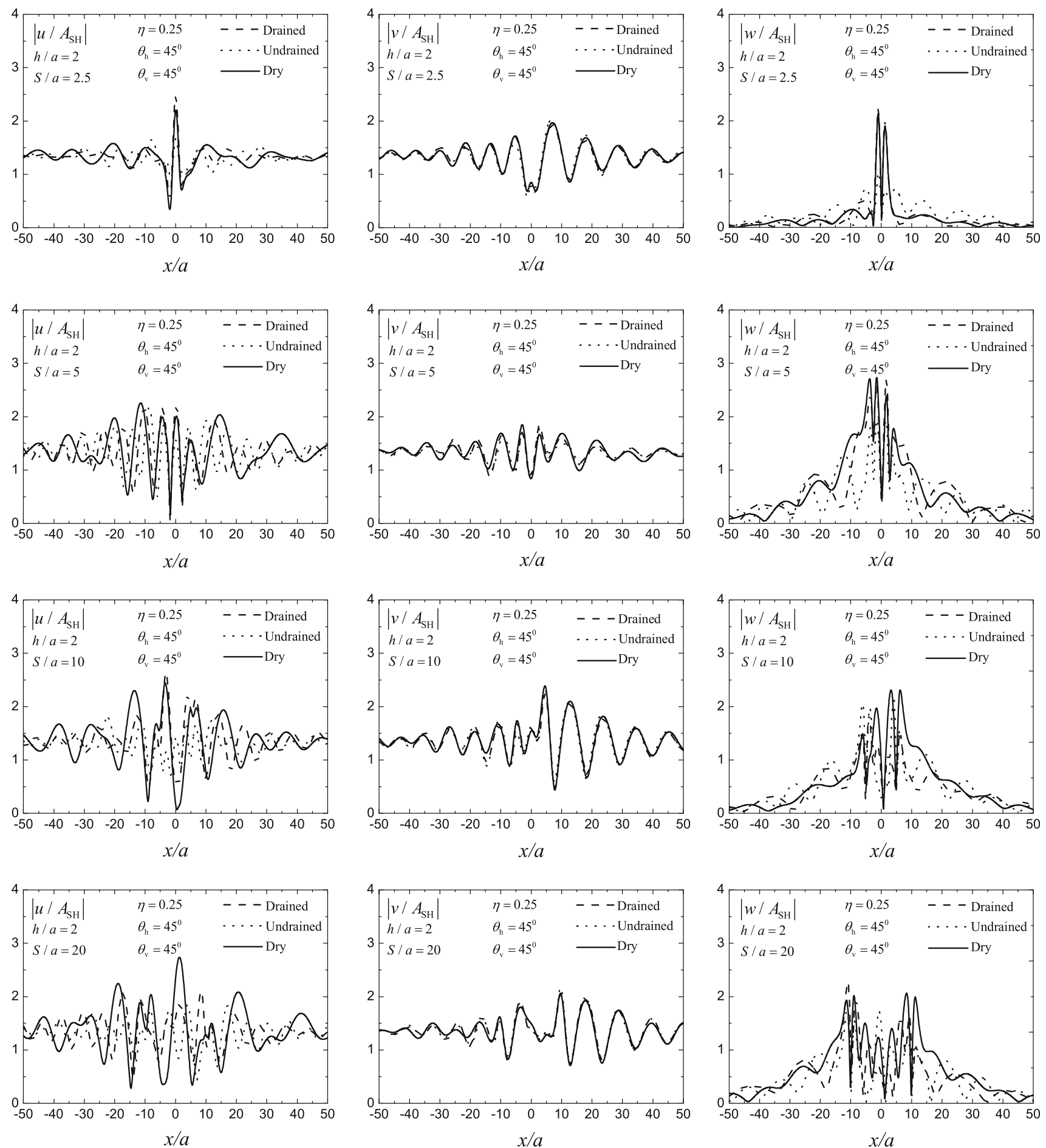

Fig. 6 Surface displacement around twin cavities in the case of dry poroelastic soil layer, the cases of saturated poroelastic soil layer with drained and undrained boundaries for $\eta=0.25$

It should be noted that, the vertical ( $\left.z^{-}\right)$surface displacement of the free filed under incident plane SH waves is zero (Fig. 10), and all the vertical (z-) surface displacements around twin cavities are resulted from the diffraction of incident waves by twin cavities. It is found that, in the case of $\eta=0.125$ the vertical displacement peaks are 3.43 (for $S / a=2.5$ ) and 1.42 (for $S / a=20$ ), respectively, and the former value is even larger than the amplification (relative 

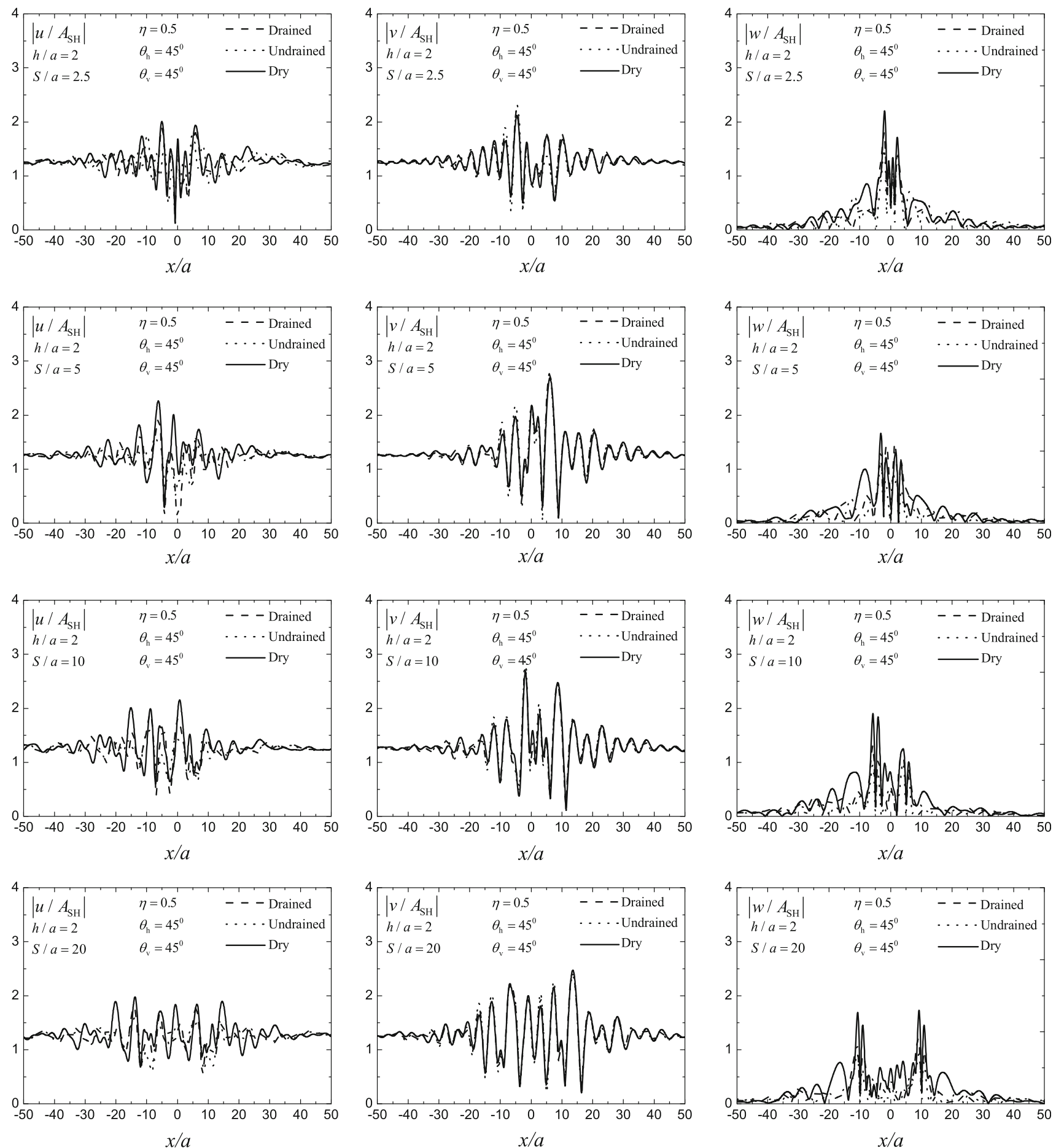

Fig. 7 Surface displacement around twin cavities in the case of dry poroelastic soil layer, the cases of saturated poroelastic soil layer with drained and undrained boundaries for $\eta=0.5$

to the free-field response) at two horizontal directions, if the free-field amplification is excluded, which shows that the wave diffraction around twin cavities is significant.

It can also be found that, there are distinct differences for surface displacement among the cases of dry poroelastic soil layer, and saturated poroelastic layer with drained and undrained boundaries, and sometimes the displacement peaks in the dry case are larger, and sometimes those in the drained case are larger, and sometime those in the undrained case are larger. The free-field response peak at the first 

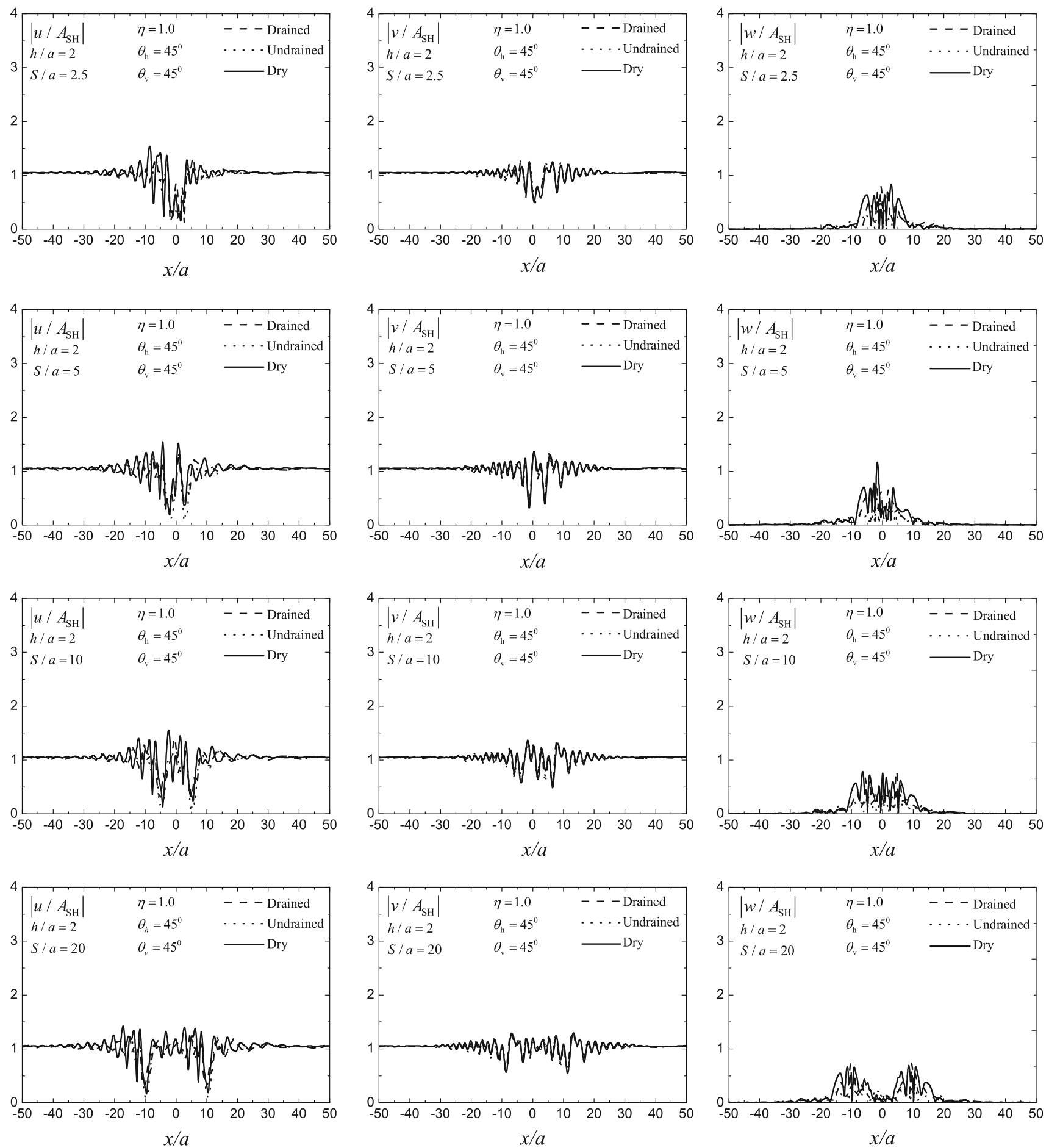

Fig. 8 Surface displacement around twin cavities in the case of dry poroelastic soil layer, the cases of saturated poroelastic soil layer with drained and undrained boundaries for $\eta=1.0$

resonant frequency in dry case is 4.51 , and the peaks in both the drained and undrained cases are 4.36. Therefore, for low frequencies, the surface displacement peaks are larger in dry case than in drained and undrained cases, since the free- field responses dominate the surface displacements; but as the incident frequency increases, the scattering, and diffraction waves play more important role, which makes the surface displacements very complicated. Under certain 

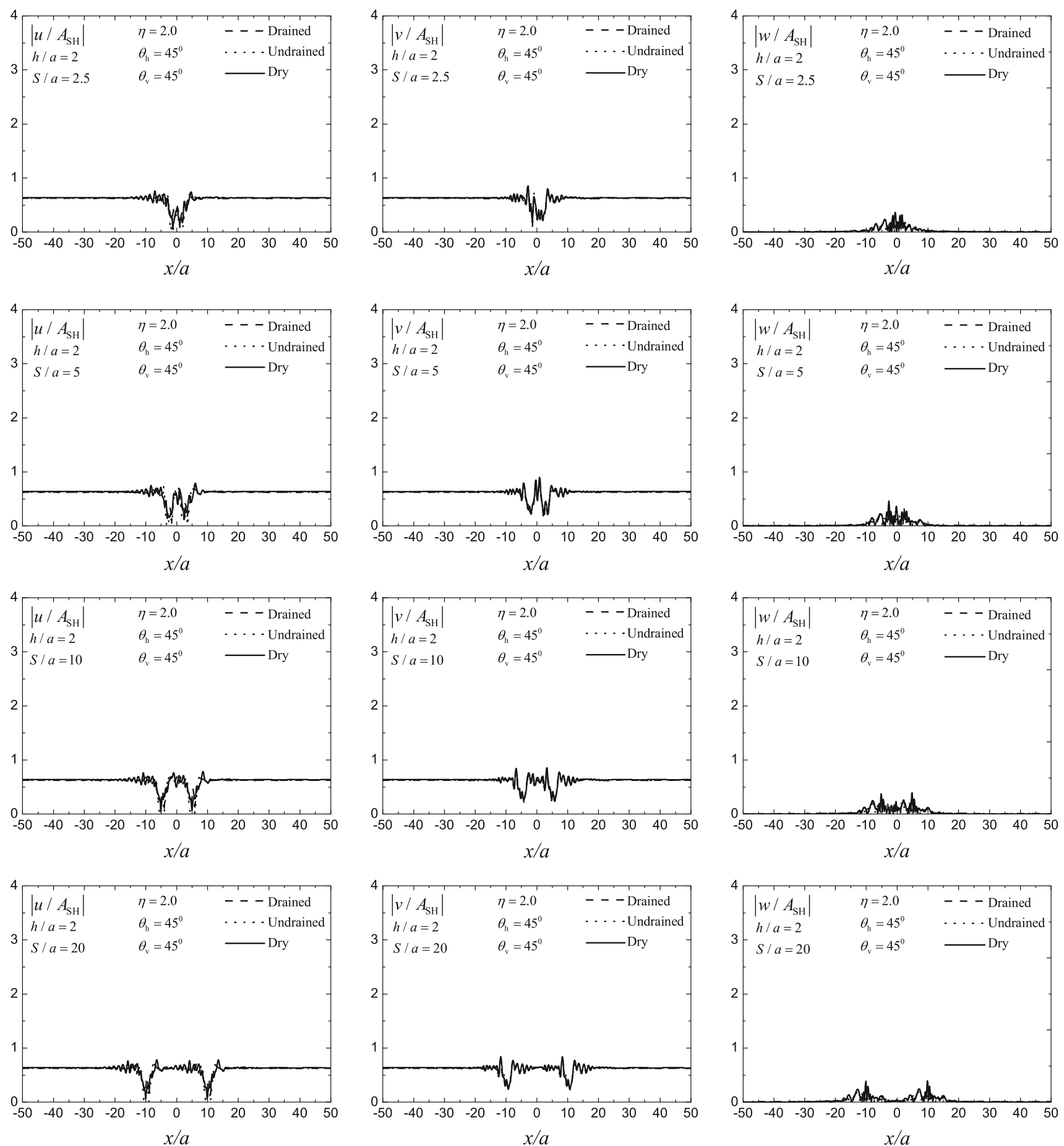

Fig. 9 Surface displacement around twin cavities in the case of dry poroelastic soil layer, the cases of saturated poroelastic soil layer with drained and undrained boundaries for $\eta=2.0$

circumstances, the differences in the case of wave diffraction among dry poroelasticity, saturated poroelasticity with drained boundary and saturated poroelasticity with undrained boundary are much larger than those in the case of free-field response, which should be paid enough attention. 

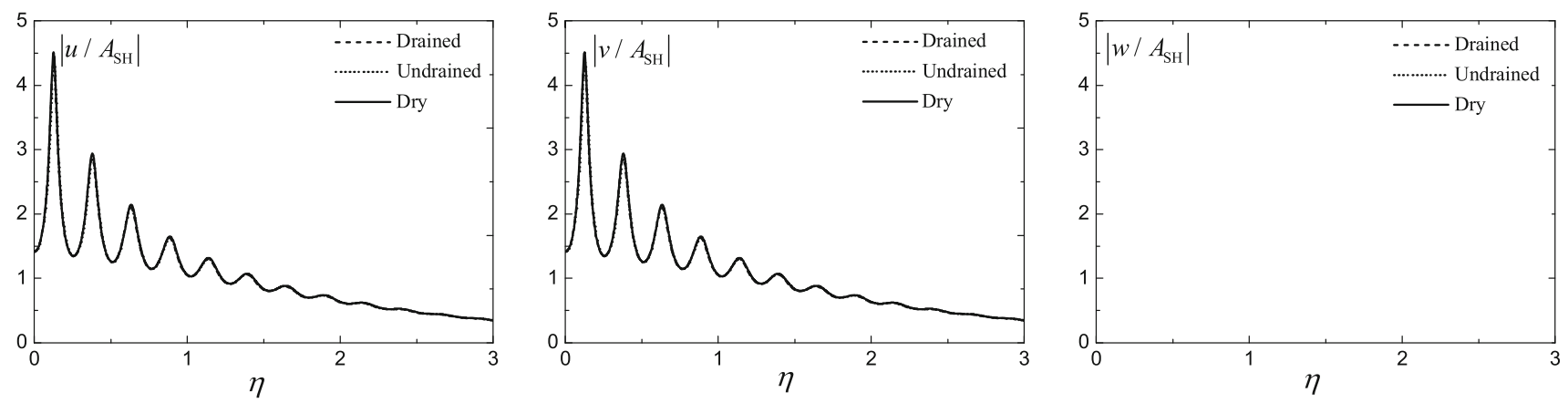

Fig. 10 Spectral amplification of surface displacement of the free field

\section{Conclusions}

3D Diffraction of obliquely incident plane SH waves by twin infinitely long cylindrical cavities in layered poroelastic half-space is studied using indirect boundary element method (IBEM), and it is shown that, the interaction between two cavities is significant and surface displacement peaks become large when two cavities are close. The influence range with large amplification of incident waves can be as wide as 40 times of the cavity radius. There exist distinct differences among the cases of dry poroelastic soil layer, and saturated poroelastic soil layer with drained and undrained boundaries under certain circumstances, and the differences may be much larger than those in the free-field response.

Acknowledgments This study is supported by National Natural Science Foundation of China under grant 51378384 and Key Project of Natural Science Foundation of Tianjin Municipality under Grant 12JCZDJC29000.

\section{References}

Biot MA (1941) General theory of three-dimensional consolidation. J Appl Phys 12(2):155-164
Biot MA (1962) Mechanics of deformation and acoustic propagation in porous media. J Appl Phys 33(4):1482-1498

De Barros FCP, Luco JE (1993) Diffraction of obliquely incident waves by a cylindrical cavity embedded in a layered viscoelastic half-space. Soil Dyn Earthq Eng 12:159-171

Liang J, Ba Z (2012) Amplification of plane SH waves by a cavity in layered half-space. J Earthq Eng Eng Vib 32(2):14-24 (in Chinese with English abstract)

Liang J, You H (2004) Dynamic stiffness matrix of a poroelastic multi-layered site and its Green's functions. Earthq Eng Eng Vib 3(2):273-282

Liang J, You H (2005) Green's functions for uniformly distributed loads acting on an inclined line in a poroelastic layered site. Earthq Eng Eng Vib 4(2):233-241

Liang J, You H, Lee VW (2006) Scattering of SV waves by a canyon in a fluid-saturated, poroelastic layered half-space, modeled using the indirect boundary element method. Soil Dyn Earthq Eng 26:611-625

Liang J, Chen J, Ba Z (2012) 3D diffraction of obliquely incident SH waves by a cylindrical cavity in layered elastic half-space (I): methodology and verification. Acta Seismol Sin 34(6):785-792 (in Chinese with English abstract)

Liang J, Chen J, Ba Z (2013) 3D diffraction of obliquely incident SH waves by a cylindrical cavity in layered elastic half-space (II): numerical results and analysis. Acta Seismol Sin 35(2):173-183 (in Chinese with English abstract)

Stamos AA, Beskos DE (1996) 3-D seismic response analysis of long lined tunnels in half-space. Soil Dyn Earthq Eng 15:111-118 